\title{
Recent Work on Strong Interactions at CERN
}

\author{
A. M. WetherelL
}

CERN, Geneva, Switzerland

$\mathrm{U}^{1}$ NLIKE the other experimental talks at this meeting, which deal with one problem or class of problems, some data are reported here, obtained since the meeting at Rochester, from three different kinds of experiments. Most of the numbers given should be regarded as provisional, either because sufficient time has not been had for good analyses of the data, or because the experiment has not been fully carried through.

First described are some data on the total cross sections, including diffraction scattering, for $p-p$ and $\bar{p}-p$ collisions at high energies. The $p-p$ cross section has been measured between 10 and $28 \mathrm{Gev} / c$ by Ashmore et al. ${ }^{1}$, and the $\bar{p}-p$ cross section at $13 \mathrm{Gev} / c$ by von Dardel et al. ${ }^{2}$ The technique of measurement of the $\bar{p}$ cross sections has been described already ${ }^{3}$ - the experiment involved a negative beam leaving the proton synchrotron at $\sim 6^{\circ}$, a high-pressure gas Čerenkov counter with a velocity resolution of about $10^{-3}$ and some beam transport. The target for the transmission measurement was $3 \mathrm{~m}$ of liquid hydrogen. The 13 $\mathrm{Gev} / c \bar{p}$ rate in a momentum interval of $6 \%$ was $\sim 1$ /synchrotron burst, and the $\tilde{p}$ content of the beam at this momentum was $\sim 0.6 \%$.

The $p$ - $p$ measurement was technically much simpler and relied on the properties of a diffraction scattered proton beam emerging at an angle of $25 \mathrm{mrad}$ from the synchrotron. The energy of this beam was changed by changing the operating energy of the accelerator and isolating the elastic peak by a magnetic selection with a $1-\mathrm{m}$ magnet. At a distance of $80 \mathrm{~m}$ from the synchrotron target, there was a proton intensity of $\sim 5 \cdot 10^{3} \mathrm{~cm}^{-2}$ per pulse at all momenta in $10-28 \mathrm{Gev} / c$. The transmission measurement was done using a $\mathrm{CH}_{2}-\mathrm{C}$ difference and counting equipment of conventional design.

Figure 1 shows the results of these experiments. Also included are some earlier data for comparison. The $p-p$ cross section appears to be constant at about $\frac{2}{3}$ of geometrical, i.e., $39-40 \mathrm{mb}$ between 10 and $28 \mathrm{Gev} / c$. Other measurements of $p$ - $p$ total cross sections at 24 $\mathrm{Gev} / c$, using visual techniques, have been made by the CERN 30-cm hydrogen bubble chamber group ${ }^{4}$ and by

${ }^{1}$ A. Ashmore, G. Cocconi, A. N. Diddens, and A. M. Wetherell, Phys. Rev. Letters 5, 576 (1960).

${ }^{2}$ G. von Dardel, R. Mermod, P. A. Piroué, M. Vivargent, G. Weber, and $\mathrm{K}$. Winter (to be published).

${ }^{3}$ G. von Dardel, D. H. Frisch, R. Mermod, R. H. Milburn, P. A Piroué, M. Vivargent, G. Weber, and K. Winter, Phys. Rev. Letters 5, 333 (1960).

${ }^{4}$ J. Bartke, R. Bock, R. Budde, W. A. Cooper, H. Filthuth, Y. Goldschmidt-Clermont, F. Grard, G. R. MacLeod, A Minguzzi-Ranzi, L. Montanet, W. G. Moorhead, D. R. O Morrison, C. Peyrou, B. W. Powell, J. Trembley and D. Wiskott (to be published). the CERN and Bern emulsion groups. ${ }^{5}$ The bubble chamber data yield a total cross section of $39.6 \pm 4.2 \mathrm{mb}$ and an elastic cross section of $12.1 \pm 3.0 \mathrm{mb}$. The emulsion group gives a total inelastic cross section of $37 \pm 11 \mathrm{mb}$ and an elastic cross section equal to $5.5 \pm 1.9$ $\mathrm{mb}$.

On looking now at the $\bar{p}-p$ cross section, we see that it falls slowly towards the $p-p$ plateau, as predicted by Pomeranchuk's theorem. Nevertheless, even at 13 Gev/c, the $\bar{p}-p$ cross section remains, at $47 \mathrm{mb}$, about $15 \%$ greater than the $p-p$ cross section at that momentum. A simple-minded decomposition of the 13 $\mathrm{Gev} / c \vec{p}-p$ cross section might be made in the following way. About $8 \mathrm{mb}$ could come from the annihilation process, i.e., the difference between the $\bar{p}-p$ and $p-p$ cross sections; of the $39 \mathrm{mb}$ left, about $10 \mathrm{mb}$ might arise from elastic scattering, which at these high energies should be similar to the $p-p$ process as it is all diffraction scattering. The inelastic cross section, i.e., that for events where the antinucleon does not annihilate, is then about $29 \mathrm{mb}$. By considering only the experimental uncertainties in these numbers, one ends up with a ratio of between about 3 and 6 for the inelastic to the annihilation cross section at $13 \mathrm{Gev} / c$.

The $K^{+}-p$ and $K^{-}-p$ cross sections have also been extended to higher energies by von Dardel et al., ${ }^{2}$ using the same setup as used for the $\bar{p}$ cross-section experiment. Some numbers of experimental interest are that at $13 \mathrm{Gev} / c$ the $K^{+}$rate was about 20 per synchrotron pulse while the $K^{-}$was about 2 . The $K^{+}$and $K^{-}$ content of the respective positive and negative beams was about $1 \%$ in each case. Figure 2 indicates the new points joining on to those which have been shown previously. ${ }^{3}$

As in the $\bar{p}-p$ and $p-p$ data, one has the feature of the antiparticle cross section, i.e., the $K^{-}$, falling down toward that of the $K^{+}$. At the highest comparable momentum, $13 \mathrm{Gev} / c$, the $K^{-}$cross section is about $25 \%$ greater than the $K^{+}$cross section. There appears to be a feeling about this data that, although the cross sections are falling slowly with energy, the difference between them is changing even more slowly. This behavior seems to be different from that of the $\pi^{+}$and $\pi^{-}$cross sections, which, as earlier reported, ${ }^{2}$ appears to be essentially the same from $4 \mathrm{Gev} / c$ up.

Now some data are presented on strange particle production, obtained by the CERN hydrogen bubble

${ }^{5}$ G. Cvijanovich, B. Dayton, P. Egli, B. Klaiber, W. Koch, M. Nikolic̀, R. Schneeberger, and H. Winzeler; J. C. Combe, W. M. Gibson, W. O. Lock, M. Schneeberger, and G. Vanderhaeghe, Nuovo cimento (to be published). 
Fig. 1. Total cross sections (including diffraction scattering) of protons and antiprotons on protons.

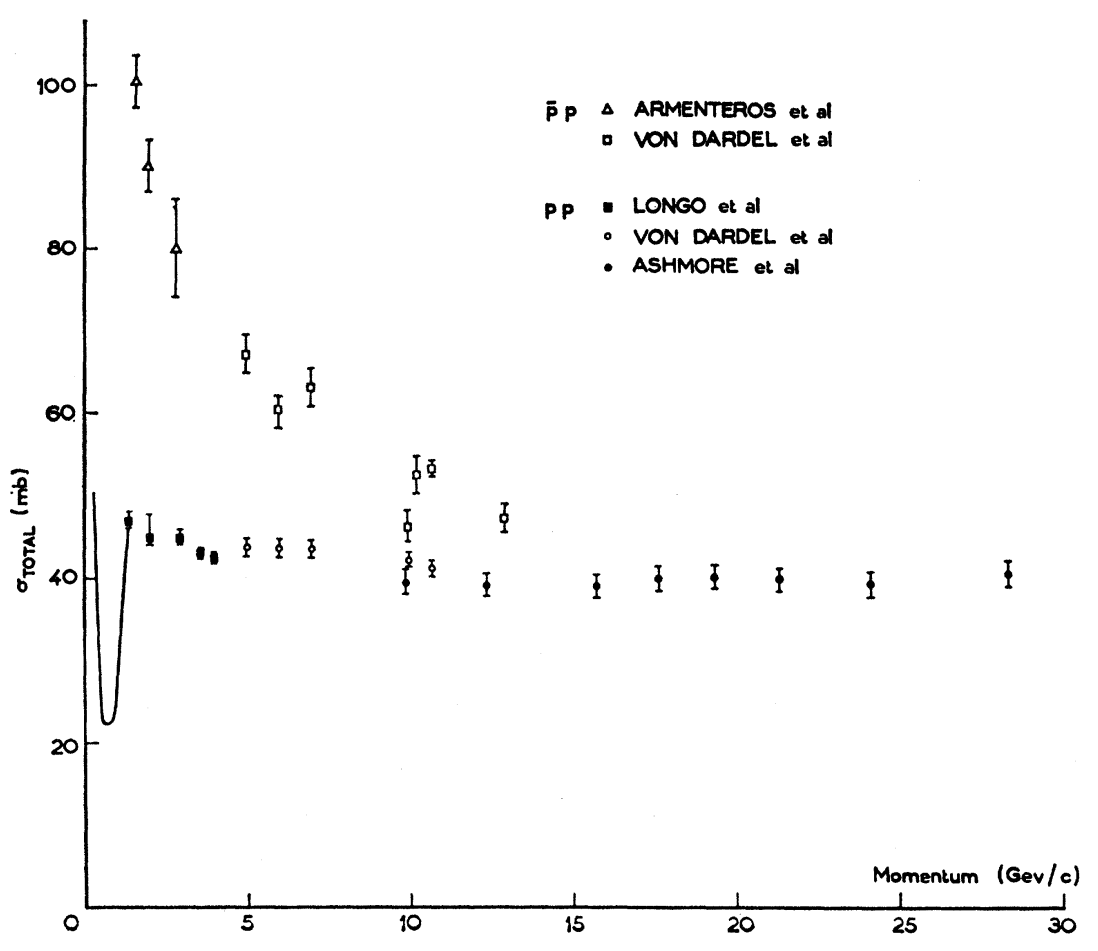

chamber group ${ }^{4}$ from jets produced by $16 \mathrm{Gev} / c \pi^{-}$ interactions in a $30-\mathrm{cm}$ liquid-hydrogen chamber.

Some preliminary results on the $\Lambda$ and neutral $K$-meson angular and momentum distributions were presented at the meeting in Rochester. We take essentially this data ${ }^{6}$ and compare it with some recently analyzed $\Sigma^{+}$and $\Sigma^{-}$from $46 V^{ \pm}$events. The most striking features of the neutral strange particle data were:

(i) the very strong backward peaking in the centerof-mass system (c.m.s.) of the hyperons, and

(ii) the essential isotropy of the neutral $K$ production. The upper half of Fig. 3 shows the angular distributions of the $\Lambda$ and of the neutral $K$ mesons. No $\Lambda$ 's in 34 cases were found to go forward and about as many $K$ 's went forward as backward. It should be noted that the $\Lambda^{\prime}$ s arose both from direct production and from $\Sigma^{0}$ decay, the experimental technique being unable to separate the two modes.

The $\Sigma^{+}$and $\Sigma^{-}$c.m.s. angular distributions from 26 and 17 events, respectively, are shown in the lower half of Fig. 3. They have a backward tendency but not nearly so sharply as the neutral hyperons, in fact, the backward to forward ratio is $\sim 5.0$ for the $\Sigma^{+}$and $\sim 2.8$ for the $\Sigma-$. These ratios were obtained by taking only $\Sigma \rightarrow \pi$ decays which should be practically unbiased. Figure 4 shows the c.m.s. momentum spectra of the strange particles. The average momentum of the $\Lambda$ and of the charged hyperons is essentially the same.

${ }^{6}$ CERN hydrogen bubble chamber group, University of Pisa, University of Trieste, Proc. Ann. Rochester Conf. High Energy Phys. 10, 402 (1960).
It is interesting to note that the mean number of charged prongs for the jets with charged strange particles is $N^{ \pm}=4.36 \pm 0.23$, essentially the same as for jets with no observable $V^{ \pm}$where $N^{ \pm}=4.3$.

A time-honored excuse ${ }^{7}$ for the backwardness of $\Lambda$ 's produced in $\pi^{-}$collisions is that there may be some sort of glancing or peripheral collision between the particles, in which a single strangeness bearing object is picked up by the nucleon, which keeps going as a hyperon into the backward cone. Present fashion might indicate the $K^{\prime},{ }^{8}$ a $(K, \pi)$ resonance, as a likely

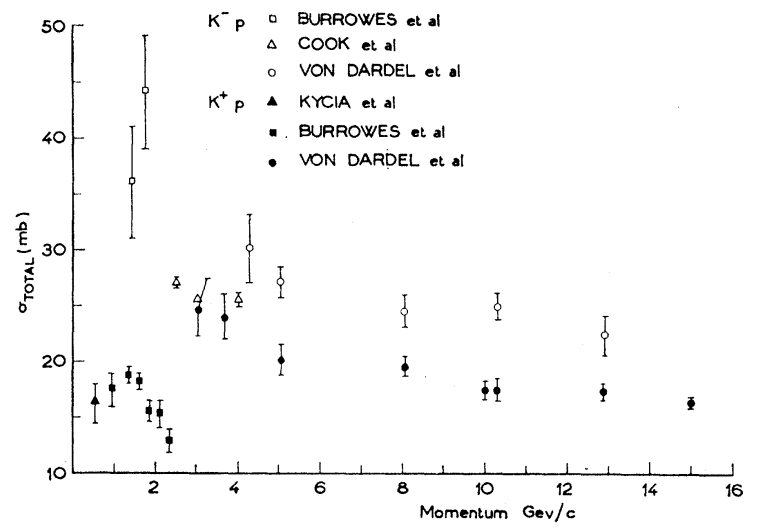

Fig. 2. Total cross sections (including diffraction scattering) of $K^{+}$and $K^{-}$on protons.

${ }^{7}$ M. Goldhaber, Phys. Rev. 101, 433 (1956); J. Schwinger, Phys. Rev. 104, 1164 (1956).

8 J. Tiomno and M. Gell-Mann, Proc. Ann. Rochester Conf. High Energy Phys. 10, 513 (1960). 

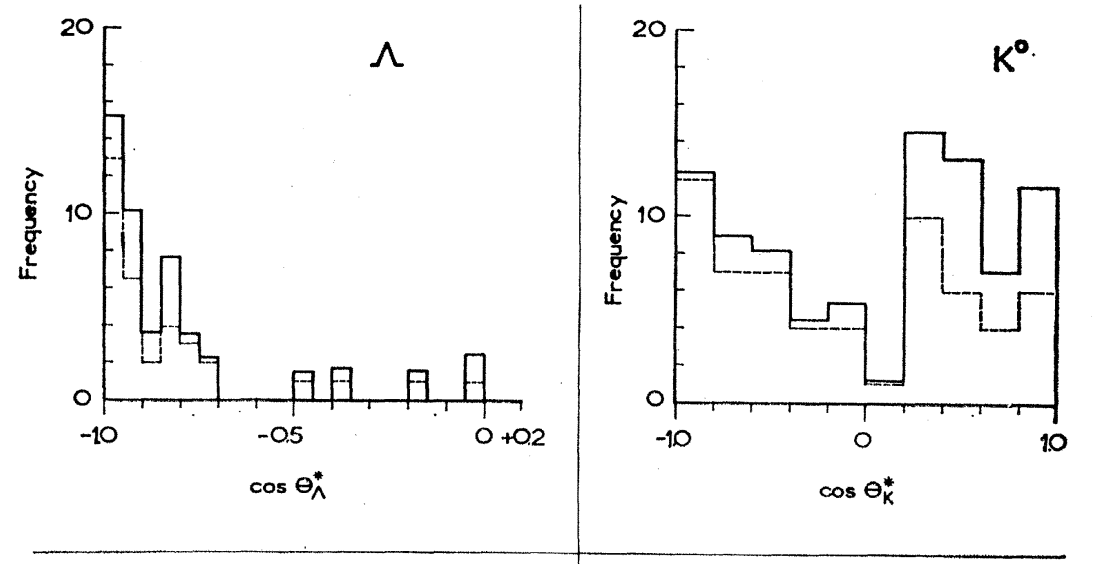

FIG. 3. Center-of-mass system angular distribution of strange particles produced in $16 \mathrm{Gev} / c^{-}$ proton interactions. The dotted
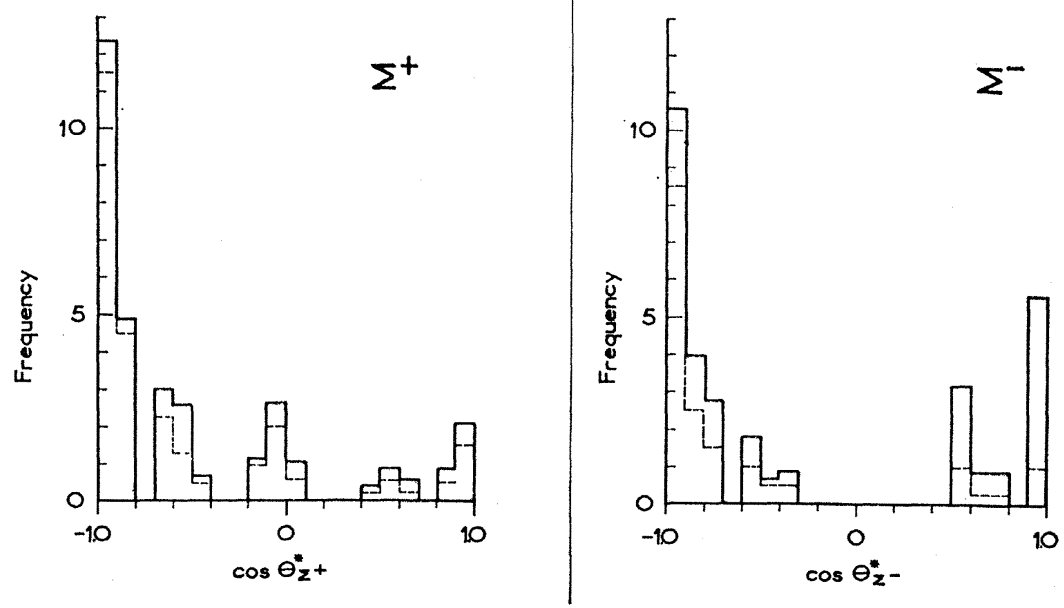
line indicates the data before correction for finite observable path length.

candidate for the exchanged object. Clearly, this sort of picture can remain valid for the $\Sigma^{+}$production, but not at all for $\Sigma^{-}$. In the latter case, the electric charge of the baryon has to suffer a change of -2 and this requires some more complicated structure in the intermediate state than a single meson exchange. Indeed, as remarked, the $\Sigma^{+}$is more peaked backwards than the $\Sigma^{-}$which might almost have the beginnings of a fore and aft distribution. As the $\Lambda$ 's are so sharply backward peaked, one might, perhaps, have expected to see some forward predominance in the $K^{0}$ distribution, but none is observed. The presence of other particles at the vertexes of a single-particle exchange process can smear out any angular correlation between the strange particles and, as the prong number shows, there were generally several other particles around, presumably pions. It is likely, also, that processes such as $K$ pair production could contribute substantially to the $K$ yield. Numbers relevant to this are that the $\Lambda$ cross section was $\sim 1 \mathrm{mb}$, while that for the neutral $K$ was $\sim 3 \mathrm{mb}$. The $\Sigma^{+}$cross section was $0.30 \pm 0.05 \mathrm{mb}$ and the $\Sigma^{-}$cross section was $0.16 \pm 0.03 \mathrm{mb}$.

Next we describe some preliminary experiments, by Cocconi, Diddens, Lillethun, and Wetherell, to study elastic and inelastic proton-nucleon scattering at high energies. The main point of interest seemed to be to study quasi-elastic processes, i.e., those in which the scattered proton retains a large fraction of its initial energy. This interest was stimulated by the shape of the momentum spectrum of protons scattered out of the synchrotron at $25 \mathrm{mrad}$ and by the work of Drell, and Salzman and Salzman, ${ }^{9}$ on the importance of peripheral collisions in quasi-elastic processes.

Figure 5 shows the momentum spectrum of the 25mrad proton beam, which was that used for the total cross-section measurements described earlier. The momentum resolution of the counting system used was $\pm 2.5 \%$. A strong inelastic tail is seen to appear on the diffraction spike. As the particles have passed through about $8 \mathrm{~cm}$ of steel and some inhomogeneous magnetic field on their way out of the accelerator, it is not reasonable to pursue the interpretation of this data very far, the trend, however, gives some sort of hint.

The stimulus from the theorists is best explained, by outlining crudely, the modified "polology" applied by Drell to inelastic nucleon-nucleon scattering. Basically, everything is shown in Fig. 6, which represents a

${ }^{9}$ S. D. Drell, Phys. Rev. Letters 5, 342 (1960); F. Salzman and G. Salzman, Phys. Rev. Letters 5, 377 (1960). 
proton-nucleon scattering by exchange of a single pion. The cross sections induced by this sort of thing near the pole are to be large for two reasons. The first is that the pion is supposed to be absorbed at vertex (b) with a cross section $\sim 30 \mathrm{mb}$ in the Gev range; secondly, the vertex (a) is not going to zero, as in the elastic case, for the incident nucleon transfers energy to the pion emitted, which then propagates with nonzero amplitude. The conditions to get near the pole are that the energy loss $\Delta$ of the scattering nucleon should be small, that is, $\Delta \equiv E_{p}-E_{p^{\prime}}<(\mu / M) E_{p}$, and that the angle of scatter should be small, $\theta_{l} \sim \mu / E_{p}$; all of the quantities here are for the laboratory system, and $E_{p}$ and $E_{p^{\prime}}$ are the incoming and outgoing energies of the scattered nucleon. For these conditions, the process shown in Fig. 6 should dominate the inelastic cross section. Finally, one can write

$$
d^{2} \sigma\left(E_{p}, E_{p^{\prime}}, \theta_{l}\right) / d \Omega d E_{p^{\prime}}=C F\left(E_{p}, \Delta, \theta_{l}\right) \sigma_{\pi N}{ }^{\operatorname{tot}}(\Delta) .
$$

One should note that the constant $C$ contains $f^{2}$, the pion-nucleon coupling constant, and if measurements should show the importance of the single-pion effect, it would, in principle, be possible to determine $f^{2}$.

As much more will be said on the theoretical aspects of this problem later in the meeting, we now discuss the rough experiments which we have set up to see if the strong yields of inelastically scattered protons, which the pole mechanism predicts, really exist.

The first experiment used a momentum analyzed $24 \pm 1 \mathrm{Gev} / c$ beam emerging from the synchrotron at $20 \mathrm{mrad}$. This beam was collimated and then hit some nucleons-in fact, a $16 \mathrm{~g} \mathrm{~cm}^{-2}$ piece of Be. The particles emerging were analyzed in direction and momentum by a system of three counters and a $2-\mathrm{m}$ long magnet. The momentum resolution of the system was $\sim \pm 5 \%$, and the momentum distributions at three angles, 10, 20, and $30 \mathrm{mrad}$, were measured. Figure 7 shows the results and, although the values of the cross sections indicated probably cannot be relied on to more than a factor of 2 or so, the shapes of the spectra are interesting. As an obvious check, the magnetic field of the spectrometer was reversed and negative particles counted. These might reasonably be assumed to be negative pions, and their intensity would presumably be the same as that of positive pions. This should therefore give a rough measurement of the pion contamination in the setup. As Fig. 7 indicates, this seemed to be negligible above $12 \mathrm{Gev} / c$. The intensity of the
FIG. 4. Center-of-mass system momentum distributions of strange particles produced in $16 \mathrm{Gev} / \mathrm{cm}^{-}$ proton interactions. The dotted line indicates the data before correction for finite observable path length.
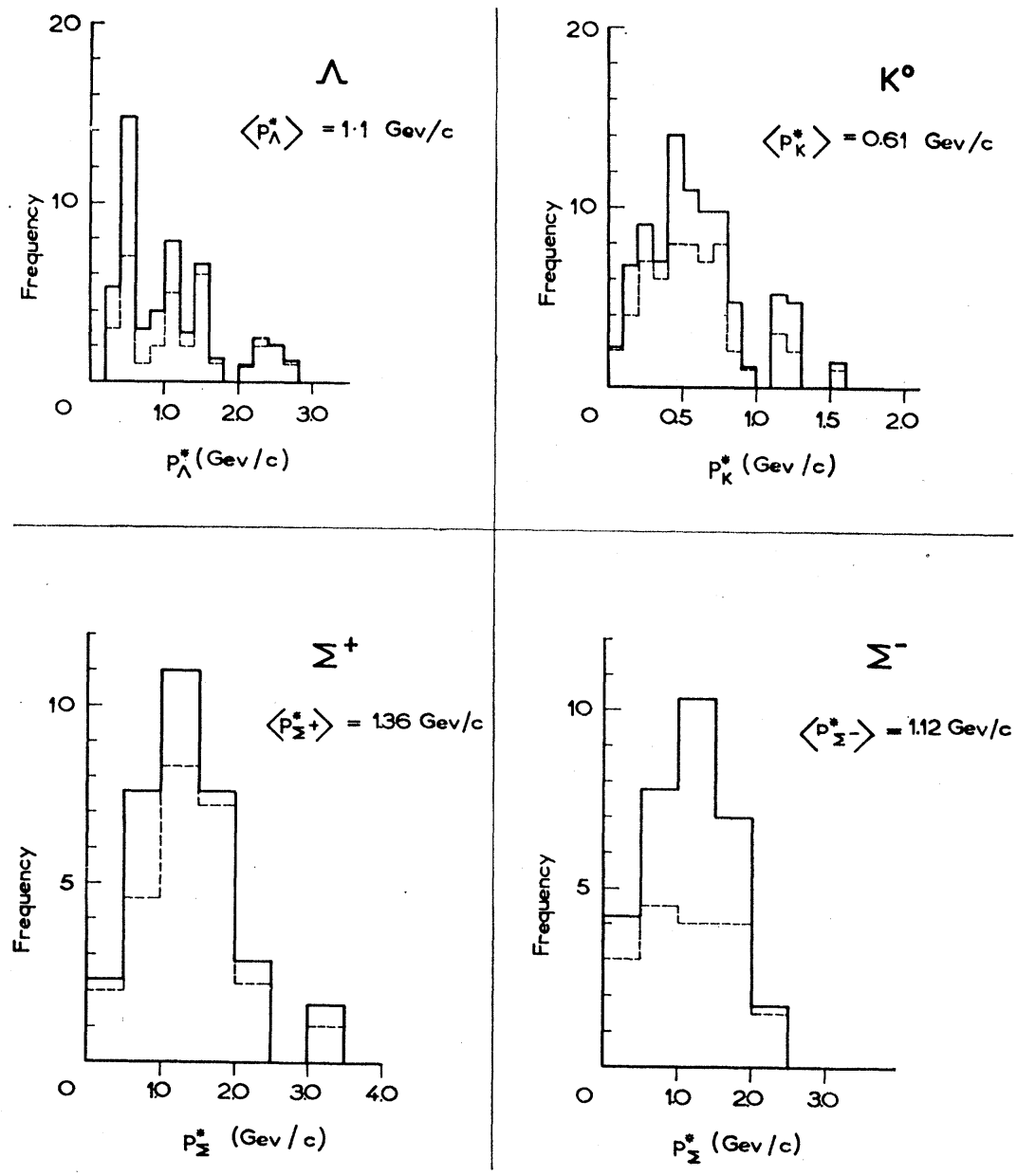


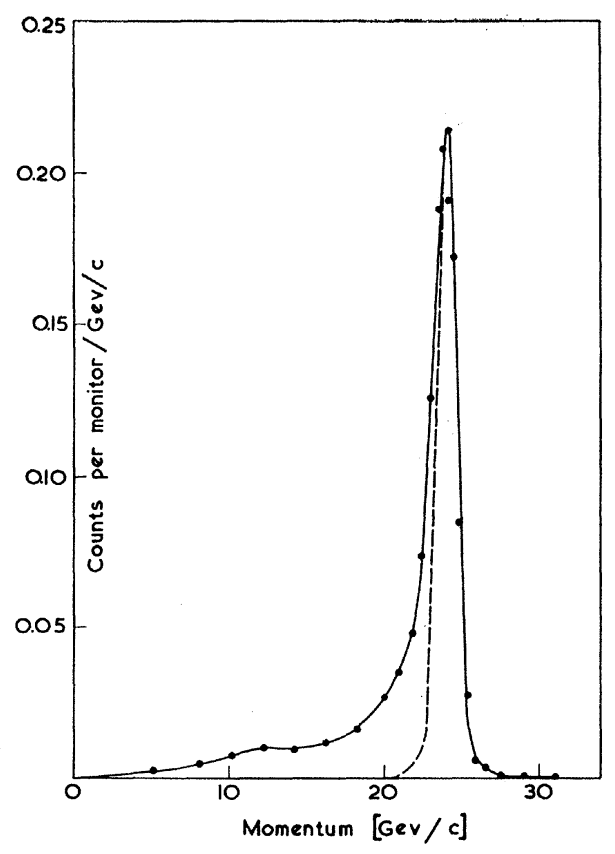

Frg. 5. Momentum spectrum of protons scattered at an angle of $25 \mathrm{mrad}$ from a Be target in the CERN proton synchrotron. The momentum resolution was about $\pm 2 \frac{1}{2} \%$.

pion yield at $5 \mathrm{Gev} / c$ was, to within a factor of 10 , in agreement with the prediction of the statistical model. ${ }^{10}$

In the proton spectra, a strong inelastic contribution is exhibited, and while the elastic contribution decreases by a factor of the order of 100 between 10 and $30 \mathrm{mrad}$, the inelastic contributions seem to vary by factors of less than 10 . The scattered proton intensity at $30 \mathrm{mrad}$ and around $20 \mathrm{Gev} / \mathrm{c}$ is $\sim 10^{3}$ times that predicted by the statistical model. ${ }^{10}$ Clearly, some violently different mechanism is at work, and the peripheral single-pion exchange process is a likely candidate. An evaluation of the formula given by Drell for the inelastic single pion pole gives cross sections $\sim 0.1 \mathrm{~b}(\mathrm{sr} \mathrm{Gev} / c)^{-1}$ at $30 \mathrm{mrad}$ between 15 and 20 $\mathrm{Gev} / c$, and $0.3-0.4 \mathrm{~b}(\mathrm{sr} \mathrm{Gev} / c)^{-1}$ between the same momenta at $20 \mathrm{mrad}$. These values are of the same order as the data in Fig. 7. One should note that the characteristic scattering angle $\theta_{l}$ is $\sim \mu / E_{p} \approx 6 \mathrm{mrad}$ and the characteristic energy loss $\Delta \sim(\mu / M) E_{p} \approx 3 \mathrm{Gev}$. Our measurements were therefore outside of this range, particularly anglewise.

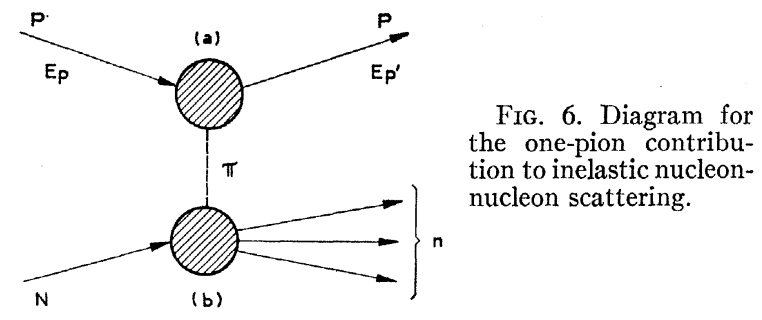

${ }^{10} \mathrm{~J}$. von Behr and R. Hagedorn, CERN 60-20 (1960).
To pursue these matters further, a more sophisticated experiment is under preparation. In this, data are taken at five angles, say 10-50 mrad, simultaneously, and a liquid-hydrogen target is used. Also, it is hoped to make the momentum resolution of the system better by using more dispersion and a better counter layout.

In the meantime, we have done some work in a situation where one can have, in principle, a system with a good momentum resolution. This is possible, provided one has sufficient numbers of particles to scatter and, indeed, inside the CERN proton synchro-

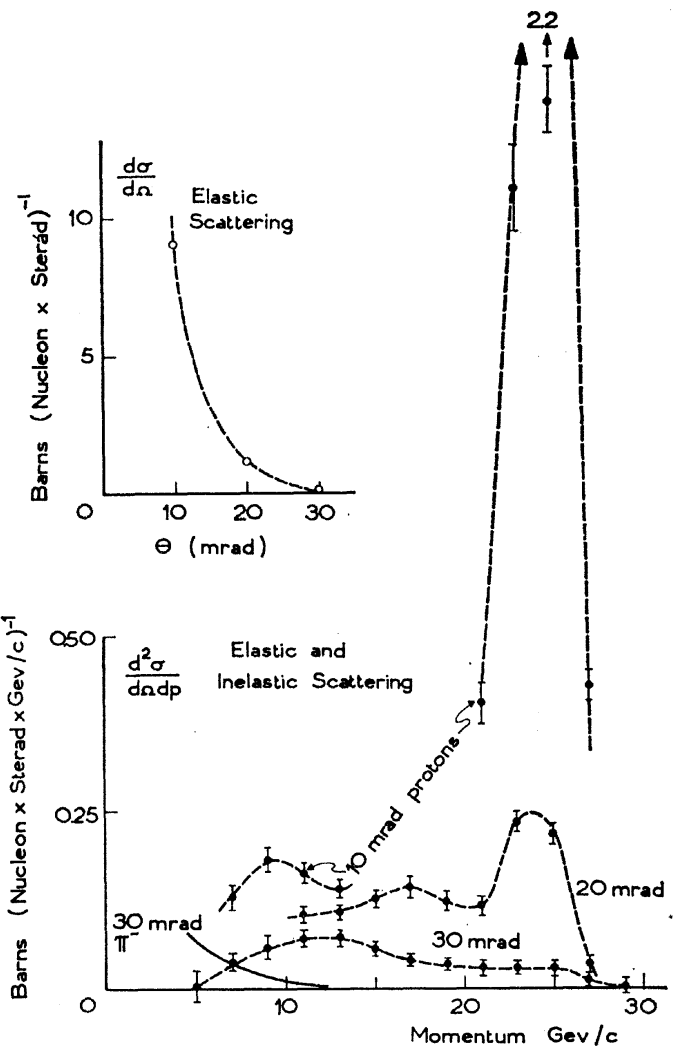

Fig. 7. Momentum spectra, at various scattering angles, for $24-\mathrm{Gev} / c$ protons on Be. The over-all momentum resolution of the analyzing system was about $\pm 5 \%$. A background equal to that indicated by detection of negative particles has been subtracted from the data. Also shown are the three points of the elastic angular distribution. The dotted line merely joins the points.

tron, there are generally about $2 \cdot 10^{11}$ protons per pulse as measured by pickup electrodes. We therefore decided to measure, with good resolution, the momentum spectrum of particles scattered out of the synchrotron by the standard 0.050 -mm-thick Be target in general use with the accelerator. In order to get the particles out of the accelerator without passing through a lot of inhomogeneous magnetic field, a large angle, $60 \mathrm{mrad}$, was chosen for taking off the scattered protons. This angle was also compatible with the emergence of the particles through a thin Mylar window, rather than through the doughnut wall, as in the case of the 25-mrad spectrum shown previously. 
Fig. 8. Momentum spectra of protons scattered at an angle of 60 mrad from a Be target in the CERN proton synchrotron. The incident momentum of the protons is indicated against each spectrum. The momentum resolution was of the order of $1 \%$.
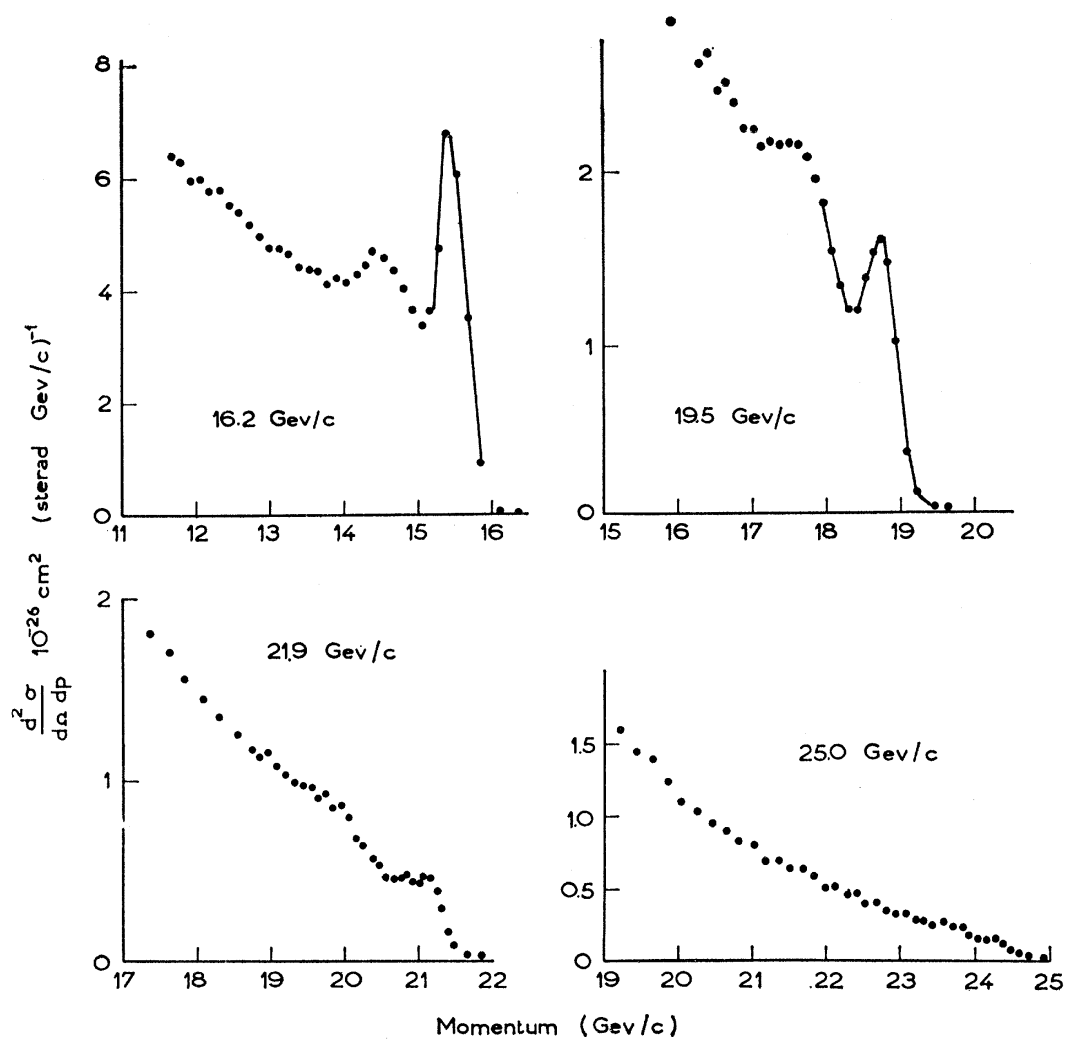

The experimental setup was then very simple. It consisted of a 6-mm-wide collimator placed about $30 \mathrm{~m}$ from the synchrotron target followed by two 2-m-long analyzing magnets and two coincidence scintillation counters, at distances of about 15 and $21 \mathrm{~m}$ and an angle of deflection of $87.5 \mathrm{mrad}$ from the magnetic center. The momentum resolving capabilities of the setup turned out to be of the order of $\pm 1 \%$.

A nice feature of the CERN proton synchrotron is the ease with which one can run at any energy between 10 and 28 Gev. On making use of this amenity, we ran momentum spectra at machine momenta of 16.2 , 19.5, 21.9, and $25 \mathrm{Gev} / c$.

The results are shown in Fig. 8. One observes a peak at the top end of the $16.2,19.5$, and $21.9 \mathrm{Gev} / c$ spectra. The position of this peak is compatible with an elastic scattering on a nucleon in the target, taking into account the recoil of the struck nucleon. The elastic peak disappears at the highest momentum $25 \mathrm{Gev} / c$. As well as the supposed elastic peak, there also appears another well-defined bump about $1 \mathrm{Gev} / c$ below.

We have looked for these phenomena in other, but unfortunately worse, experimental conditions. The momentum spectra of particles resulting at $20 \mathrm{mrad}$ for a machine momentum of $24 \mathrm{Gev} / c$ and at $40 \mathrm{mrad}$ for momenta of 16 and $24 \mathrm{Gev} / c$ have been measured and are shown in Fig. 9, which shows, strikingly, the inelastic peak again at about $1 \mathrm{Gev} / c$ below the elastic spike. For the spectra of Fig. 9, the particles passed through something of the order of $10 \mathrm{~cm}$ of stainless steel and, even worse, through a lot of inhomogeneous magnetic field in the accelerator. Only a rough analysis of these perturbations has so far been made. For this reason, and because of the uncertainties inherent in the normalization of internal beam experiments, the scale of cross sections attached to Figs. 8 and 9 was obtained by appeal to the results of the external beam experiment. In any case, the cross sections should be regarded as tentative data for the time being.

Let us now look at some properties of the inelastic peak, without regard to values of cross sections.

Table I gives the values of the four-momentum transfer $q$ and the excitation energy $M^{*}$ of the struck

TABLE I. Summary of the kinematic conditions for the experimental data in Figs. 8 and 9. The right-hand columns give the invariant four-momentum transfer $q$ for the inelastic peak and the excitation energy $M^{*}$ of the struck nucleon.

\begin{tabular}{cccccc}
\hline \hline $\begin{array}{c}\text { Incident } \\
\text { proton } \\
\text { momentum } \\
p(\mathrm{Gev} / c)\end{array}$ & $\begin{array}{c}\text { Angle } \\
(\mathrm{mrad})\end{array}$ & $\begin{array}{c}\text { Momentum } \\
\text { at elastic } \\
\text { peak } \\
(\mathrm{Gev} / c)\end{array}$ & $\begin{array}{c}\text { Momentum } \\
\text { at inelastic } \\
\text { peak } p^{\prime} \\
(\mathrm{Gev} / c)\end{array}$ & $\begin{array}{c}q \\
\mathrm{Gev} / c\end{array}$ & $\begin{array}{c}M^{*} \\
\mathrm{Gev}\end{array}$ \\
\hline 24.2 & 20 & 23.9 & 23.1 & 0.47 & 1.65 \\
16.0 & 40 & 15.8 & 14.8 & 0.62 & 1.66 \\
24.2 & 40 & 24.0 & 23.0 & 0.94 & 1.50 \\
16.2 & 60 & 15.5 & 14.5 & 0.92 & 1.80 \\
19.5 & 60 & 18.6 & 17.5 & 1.11 & 1.85 \\
21.9 & 60 & 20.8 & $\approx 19.8$ & 1.25 & 1.81 \\
\hline \hline
\end{tabular}



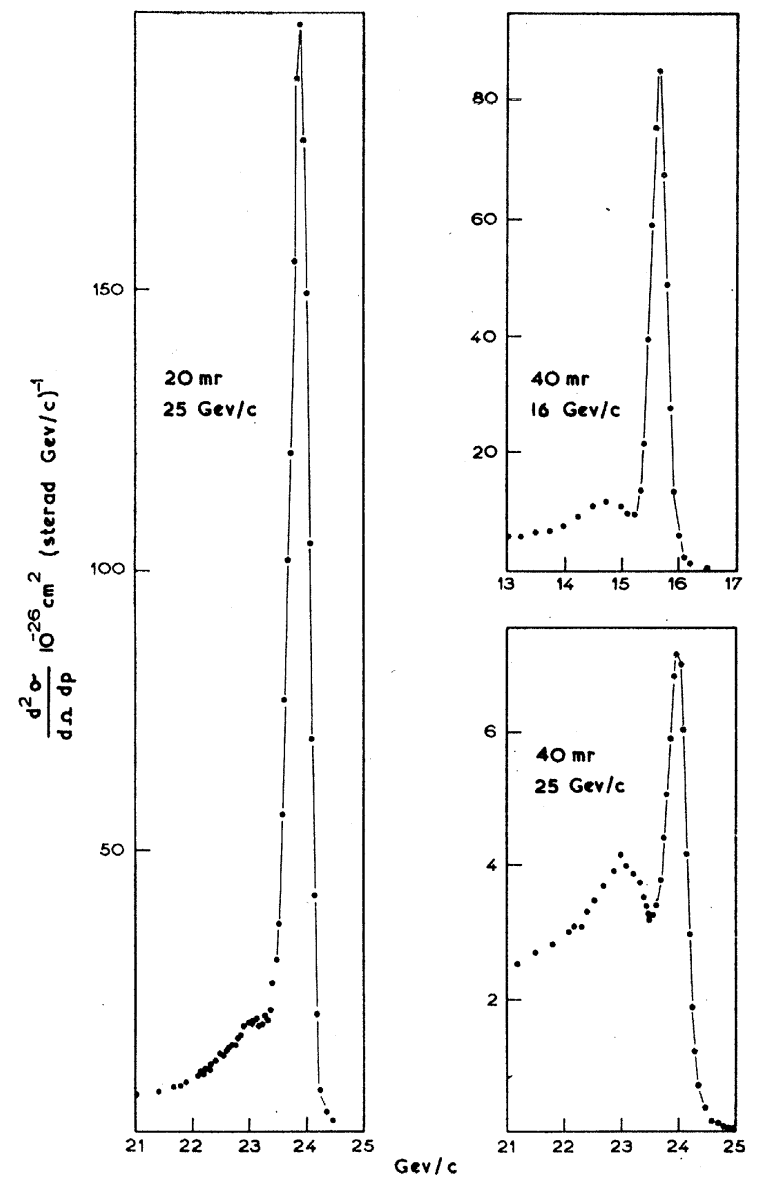

FIG. 9. Momentum spectra, at various angles and incident momenta, of protons scattered out of the CERN proton synchrotron by a Be target.

nucleon at the inelastic peak for the various experimental conditions.

The two quantities $q$ and $M^{*}$ are given by the following equations:

$$
\begin{aligned}
q^{2} & =\left(\mathbf{p}^{\prime}-\mathbf{p}\right)^{2}-\left(E_{p^{\prime}}-E_{p}\right)^{2} \\
M^{* 2} & =\left(M+E_{p}-E_{p^{\prime}}\right)^{2}-\left(\mathbf{p}-\mathbf{p}^{\prime}\right)^{2},
\end{aligned}
$$

where $\mathbf{p}$ and $\mathbf{p}^{\prime}$ are the initial- and final-state laboratory system momenta of the scattering proton. It is interesting to observe that, while the four-momentum transfer $q$ changes by almost a factor of 3 over the range $0_{1}$ kinematics studied, the value for $M^{*}$ is essentially constant. As indicated by the external beam experiment, the yield of protons at small inelasticities $(\sim 5 \%)$ was at least of the order of a factor $10^{3}$ times greater than a statistical theory could predict.

The most reliable data were obtained at $60 \mathrm{mrad}$, and it is not clear as to whether there is any validity in a direct comparison with Drell's evaluation, where the angles involved may be $\sim 10 \mathrm{mrad}$. Perhaps one can say that qualitatively the high yield of inelastically scattered particles is in accord with the peripheral collision picture outlined earlier. The origin of the inelastic peak remains unexplained. One notes that the energies $M^{*}$ appearing in Table $I$ are in the region of the total c.m.s. energies of the second and third resonance in $\pi^{-}$ proton interactions and of the high-energy bump in the $\pi^{+}$proton total cross section. The latter energies are $1.52,1.67$, and $1.92 \mathrm{Gev}$, respectively.

It would not seem easy, however, to explain the observed features of the inelastic peak on the basis of Fig. 6. An apparently important feature of the data is that the inelastic peak is observed only when the diffraction peak is present. This point possibly reflects some connection between the inelastic peak and the diffraction process. ${ }^{11}$ It is obvious that much experimental work is indicated for a clarification of the problems raised by these simple experiments.

I am indebted to colleagues working on experiments with the CERN proton synchrotron who have spent time discussing their data with me. For attendance at the Conference, I acknowledge with gratitude financial support of the American Institute of Physics and the kind hospitality of the Department of Physics at Berkeley.

${ }^{11}$ Note added after the Conference: Our attention has been drawn, by $\mathrm{L}$. van Hove, to diffraction disintegration processes [cf. M. L. Good and W. D. Walker, Phys. Rev. 120, 1857 (1960) and earlier work referred to therein]. It may be possible to explain the inelastic peak by diffraction disintegration of the target nucleons. On applying the arguments discussed by Good and Walker (for the nucleon-nucleus case) to the nucleon-nucleon interaction, it should be expected that diffraction dissociation of the target nucleon into a nucleon and a pion would occur, in our conditions, for energy transfers of $\sim 1 \mathrm{Gev}$. The fact that the inelastic peak follows the diffraction peak results naturally. So far, it seems difficult to make these ideas quantitative.

\section{DISCUSSION}

E. Segrè, University of California, Berkeley, California: How much of your inelastic scattering can be attributed to your use of beryllium, and not hydrogen?

A. M. Wetherell: This is not known. One of our next objects is to study this question with hydrogen, but before doing that we shall look to see if this sort of structure changes with different targets inside the synchrotron. We shall change from $\mathrm{Be}$ to $\mathrm{Pt}$, or something of that sort. I feel that as one has observed an elastic peak at a momentum difference corresponding to nucleon-nucleon events one is really looking at nucleon-nucleon phenomena.
J. Bernstein, Brookhaven National Laboratory, Upton, New York: Three brief remarks which have to do with this strangeness bearing thing going across and accounting for your angular distribution: Firstly, the same strangeness bearing thing would probably predict that the $\Sigma^{0}$ would also be peaked backwards. That is something to look for.

Secondly, if you measure the angular distribution of the $K$ 's which are produced in the reaction $\pi+p \rightarrow K+\Lambda+$ pions, you would expect the angular distribution for the $K$ 's when the pion multiplicity is small to be strikingly different from that of the $K$ 's when the pion multiplicity is large, because it is 
likely that the $K$ and the $\pi$ are associated in some sort of resonant structure; in fact, the Russians seem to have found this at $6.8 \mathrm{Bev}$.

Thirdly, if you take this picture seriously, it is not easy to account for the large polarization of the $\Lambda^{0}$. Therefore, one should see whether the large $\Lambda^{0}$ polarization remains at these high energies, and if so, I think that this particular interpretation of the angular distributions requires more refinement.

A. M. Wetherell: Yes, I agree with everything you say. With the small hydrogen bubble chamber it was impossible to distinguish between directly produced $\Lambda$ and $\Lambda$ coming from $\Sigma^{0}$ decay.

L. Alvarez, University of California, Berkeley, California:
I have an experimental comment on the remarks just made about the angular distribution of the lambda's. The $30-\mathrm{cm}$ hydrogen chamber has an effective diameter for decay between 10 and $12 \mathrm{~cm}$. That is the potential path. The mean decay distance for a forward produced $\Lambda$ in this energy range is approximately $200 \mathrm{~cm}$, which makes the effective length of the bubble chamber for this class of events quite small. Have any corrections been made for this? I think the backward peaking is very reasonable, but this instrumental effect introduces a bias.

A. M. Wetherell : Your concern seems valid. Peyrou's group labored at this and they concluded that they should have seen some forward going $\Lambda$. This was taken into account.

\title{
$K$-Nucleon Interaction
}

\author{
LEROY T. KERTH \\ Lawrence Radiation Laboratory, University of California, Berkeley 4, California
}

\section{INTRODUCTION}

$T^{n}$ HIS paper summarizes the broad details of the $K$-nucleon interaction and presents data from three new experiments carried out at the Lawrence Radiation Laboratory. These new experiments are: I. the total cross section for $K^{-}$mesons on protons and neutrons in the momentum region ${ }^{1} 1-4 \mathrm{Bev} / c$; II. total cross section for $K^{-}$mesons on protons and neutrons in the momentum region ${ }^{2} 0.6-1.1 \mathrm{Bev} / c$; and III. total cross section for $K^{+}$mesons on protons and neutrons in the momentum region ${ }^{3} 0.8-2.9 \mathrm{Bev} / c$. In the final section we speculate on some possible interpretations of the data as they appear at the present time. Since the analysis and correction of the data from experiments II and III have not been completed yet, the corresponding results are presented below with rather large errors.

\section{II. $K^{-}$-NUCLEON TOTAL CROSS SECTION}

There are many accurate total cross-section measurements now available for $K^{-}$mesons on pr itons up to a momentum of $10 \mathrm{Bev} / c$. These data are sisown in Figs. 1 and 2. Figure 1 shows the total cross section as a function of laboratory momentum between 1 and $9 \mathrm{Bev} / c$. The data from experiment I and the data from von Dardel ${ }^{4}$ are consistent and indicate that the cross section above $3 \mathrm{Bev} / c$ is essentially flat with momentum at a value of

${ }^{1}$ V. Cook, B. Cork, T. F. Hoang, D. Keefe, L. T. Kerth, W. A. Wenzel, and 'T. F. Zipf (unpublished).

${ }^{2}$ O. Chamberlain, K. M. Crowe, D. Keefe, L. T. Kerth, A Lemonick, T. Maung, and T. F. Zipf (to be published).

3 V. Cook, D. Keefe, L. T. Kerth, P. G. Murphy, W. A. Wenzel, and $\mathrm{T}$. F. Zipf (to be published).

${ }^{4}$ G. von Dardel, D. H. Frisch, R. Mermod, R. H. Milburn, P. A. Piroué, M. Vivargent, G. Weber, and K. Winter, Proc. Ann. Rochester Conf. High Energy Phys. 10, 484 (1960). approximately $25 \mathrm{mb}$. Figure 2 shows the $K^{-}$total cross section from 0 to $3 \mathrm{Bev} / c$. The data from experiment II show a considerable structure (which we tentatively refer to as a "resonance") in the $K^{-}$-proton cross section in the region of $1 \mathrm{Bev} / c$. The resonance, however, does not appear - at least to anything like the same degreein the $K^{-}$-neutron cross section, also shown in Fig. 2. Since the $K^{-}$-neutron system is a pure $T=1$ state and the $K^{-}$-proton system is a mixture of the $T=1$ and $T=0$ states, the resonance in the $K^{-}$-proton cross section is in the $T=0$ state. The structure evident in the $K^{-}$-proton cross section in the region $1.5 \mathrm{Bev} / c$ in Figs. 1 and 2 is of smaller magnitude and needs to be investigated more thoroughly. A better idea as to the position and the shape of the resonance at $1 \mathrm{Bev} / c$ can

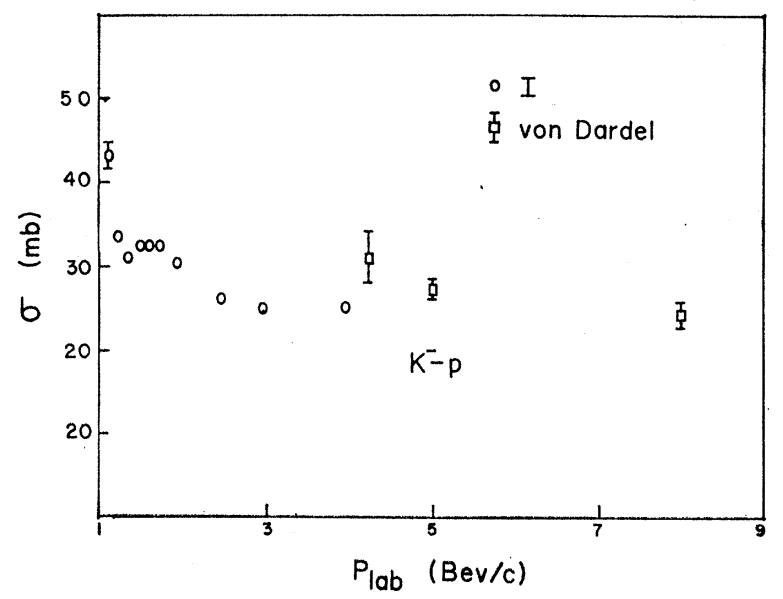

Fig. 1. $K^{-}-p$ total cross section in the momentum range $1-4$ $\mathrm{Bev} / c$. For the data of experiment I where no error bars are shown, the errors are smaller than the symbols used. 